\title{
Hot and cold running water: understanding evolved star winds
}

\author{
A. M. S. Richards ${ }^{1}$, M. D. Gray ${ }^{1}$, A. Baudry ${ }^{2}$, E. M. L. Humphreys ${ }^{3}$, \\ S. Etoka ${ }^{1}$, L. $\operatorname{Decin}^{4}$, I. Marti-Vidal ${ }^{5}$, A. M. Sobolev ${ }^{6}$ and \\ W. Vlemmings ${ }^{5}$ \\ ${ }^{1}$ JBCA, School of Physics \& Astronomy, University of Manchester, M13 9PL, UK \\ contact email: amsr@jb.man.ac.uk \\ ${ }^{2}$ Laboratoire d'astrophysique de Bordeaux, Univ. Bordeaux, CNRS, B18N, F-33615 Pessac, \\ France ${ }^{4}$ ESO Karl-Schwarzschild-Str. 2, 85748 Garching, Germany \\ ${ }^{4}$ Instituut voor Sterrenkunde, Katholieke Universiteit Leuven, 3001 Leuven, Belgium \\ ${ }^{5}$ Dept. of Earth, Space and Environment, Chalmers University of Technology, Onsala Space \\ Observatory, SE 43992 Onsala, Sweden ${ }^{6}$ Ural Federal University, Ekaterinburg, Russia
}

\begin{abstract}
Outstanding problems concerning mass-loss from evolved stars include initial wind acceleration and what determines the clumping scale. Reconstructing physical conditions from maser data has been highly uncertain due to the exponential amplification. ALMA and eMERLIN now provide image cubes for five $\mathrm{H}_{2} \mathrm{O}$ maser transitions around VY CMa, at spatial resolutions comparable to the size of individual clouds or better, covering excitation states from 204 to $2360 \mathrm{~K}$. We use the model of Gray et al. 2016, to constrain variations of number density and temperature on scales of a few au, an order of magnitude finer than is possible with thermal lines, comparable to individual cloud sizes or locally almost homogeneous regions. We compare results with the models of Decin et al. 2006 and Matsuura et al. 2014 for the circumstellar envelope of VY CMa; in later work this will be extended to other maser sources.
\end{abstract}

Keywords. masers, stars: late-type, stars: winds, outflows

\section{Water maser measurements and modelling}

The ideal observation of bright circumstellar $\mathrm{H}_{2} \mathrm{O}$ masers uses velocity channels $\leqslant 0.1$ $\mathrm{km} \mathrm{s}^{-1}$ at an angular resolution $\theta_{\mathrm{B}}$ of $10-20$ mas, sensitive to scales up to $\sim 100$ mas. This detects, and resolves, all the 22-GHz emission from Asymptotic Giant Branch (AGB) and Red Supergiant (RSG) stars at $\geqslant 0.1$ and $1 \mathrm{kpc}$, respectively. The channel sampling provides arbitrary slices through the velocity dispersion or gradient in each maser cloud. The position and size $s$ of each maser spot can be found with an accuracy $\sim \theta_{\mathrm{B}} /$ (signalto-noise ratio), for sparse visibility plane coverage. $s$ is the beamed size, but the parent cloud size $L$ can be estimated from the total angular separation of nearby components in adjacent channels, used to find the beaming angle $\Omega=s^{2} / L^{2}$ (Richards et al. 2011). This distinguishes between $\sim$ spherical maser clouds quiescently expanding in the outflow, or shocked slabs and other structures elongated along the line of sight; the brightest masers have smaller spots in the former case whilst in the latter the spot size increases up to the parent cloud size (Elitzur et al. 1991). These measurements are possible using e.g. (e-) MERLIN or ALMA $\dagger$ on long baselines; whilst the higher resolution of VLBI is superb for proper motion measurements it usually resolves-out much emission. The ALMA Science

$\dagger$ ALMA is a partnership of ESO, NSF (USA) and NINS (Japan), together with NRC (Canada), MOST and ASIAA (Taiwan), and KASI (Republic of Korea), in cooperation with the Republic of Chile. The Joint ALMA Observatory is operated by ESO, AUI/NRAO and NAOJ. 

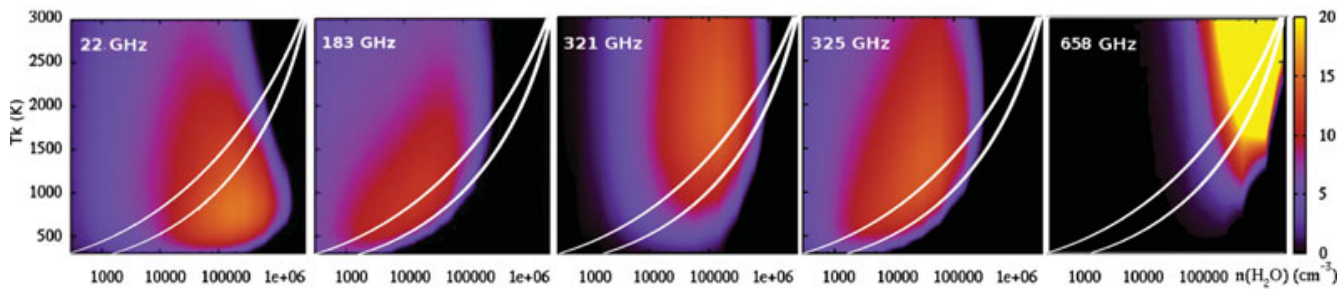

Figure 1. Maser optical depth as a function of $o-\mathrm{H}_{2} \mathrm{O}$ number density and $T_{\mathrm{k}}$. The white lines enclose likely conditions in a CSE, from the star at the top right of each panel.

Table 1. Frequencies and upper energy levels of $\mathrm{H}_{2} \mathrm{O}$ maser lines imaged around evolved stars. The spin is given; all are ground vibrational state unless given.

\begin{tabular}{|l|ccccc|}
\hline Line $(\mathrm{GHz})$ & 22.235079 & 183.31012 & 321.22564 & 325.15292 & 658.00655 \\
State etc. & $o$ & $p$ & $o$ & $p$ & $o, v 2=1$ \\
$E_{\mathrm{U}}(\mathrm{K})$ & 643 & 200 & 1861 & 454 & 2360 \\
\hline
\end{tabular}

Verification (SV) results (and MERLIN data for VY CMa, owing to its low declination) presented here have $\theta_{\mathrm{B}} \geqslant 90$ mas, resolving clouds but not individual spots.

$22-\mathrm{GHz}$ masers around effectively solitary stars are found in $\approx$ spherical shells from $\sim 5$ to $\sim 20 R_{\star}$ (about 1 (5) au for AGB (RSG)). The wind shows gradual, radial acceleration, achieving escape velocity during its passage through the $22-\mathrm{GHz}$ shell. Individual cloud are of order 1.5 (15) au around AGB (RSG), consistent with radial expansion from a cloud birth size $\sim 0.1 R_{\star}$ at the stellar surface. The $22-\mathrm{GHz}$ cloud filling factor is $<1 \%$ but the inner radius, determined by the maser quenching density (Cooke \& Elitzur 1983), suggests that they are $10-100 \times$ overdense, i.e. most of the mass loss is concentrated in a few clouds ejected per stellar pulsation cycle (Richards et al. 2012).

Gray et al. (2016) comprehensively models of $\mathrm{H}_{2} \mathrm{O}$ masers in CSEs (circumstellar envelopes; see that paper for additional references). This predicts $40-50 \mathrm{H}_{2} \mathrm{O}$ maser lines in ALMA bands at energy levels $\left(E_{\mathrm{U}}\right) \leqslant 7200 \mathrm{~K}$, arising from distinct combinations of dust and gas temperatures $\left(300<T_{\mathrm{K}}<3000 \mathrm{~K}\right)$, gas number density $\left(10^{5}<n<10^{11} \mathrm{~cm}^{-3}\right)$, $\mathrm{H}_{2} \mathrm{O}$ fractional abundance $\left(f_{\mathrm{nH} 2 \mathrm{O}}\right)$, velocity and radiation fields. About 16 of these have been detected spectrally, but only 5 have published images, all (apart from $22 \mathrm{GHz}$ ) for VY CMa (Richards et al. 2014; Marti-Vidal et al. 2016). These are listed in Table 1. Fig. 1 shows their predicted (negative) maser optical depth as a function of $o-\mathrm{H}_{2} \mathrm{O}$ number density and gas kinetic temperature, assuming the ortho:para ratio $(o: p)=3: 1$ and $f_{\mathrm{nH} 2 \mathrm{O}}=3 \times 10^{-5}$. This shows that these lines sample a variety of physical conditions.

\section{VY CMa water maser observation-model comparison}

The RSG VY CMa lies at $1.1 \mathrm{kpc}$ (Choi et al. 2008), with a $2.2 \mu \mathrm{m}$ stellar radius $R_{\star}$ of 5.7 mas (Wittkowski et al. 2012), and an $L S R$ velocity $V_{\star}=22 \mathrm{~km} \mathrm{~s}^{-1}$. The 183,321 , 325 and $658 \mathrm{GHz}$ masers were observed for ALMA Science Verification in 2016 and 2013 (Marti-Vidal et al. 2016; Richards et al. 2014) The star was found to be at the centre of expansion and used to align the data sets. The 22-GHz masers used here were observed in 1994 (Richards et al. 1998) and 2000 using MERLIN and in 2016 as e-MERLIN test observations. The 22-GHz maser Doppler velocities and proper motions (including comparison with Bowers et al. 1993) show that, between $\sim(70-450)$ mas radius, the wind is accelerating radially away from the star. However, VY CMa has a highly irregular CSE on arcsec scales, with no axis of symmetry, and HST line observations suggest clumps are ejected along curved, apparently ballisic trajectories (Humphreys et al. 2007). 

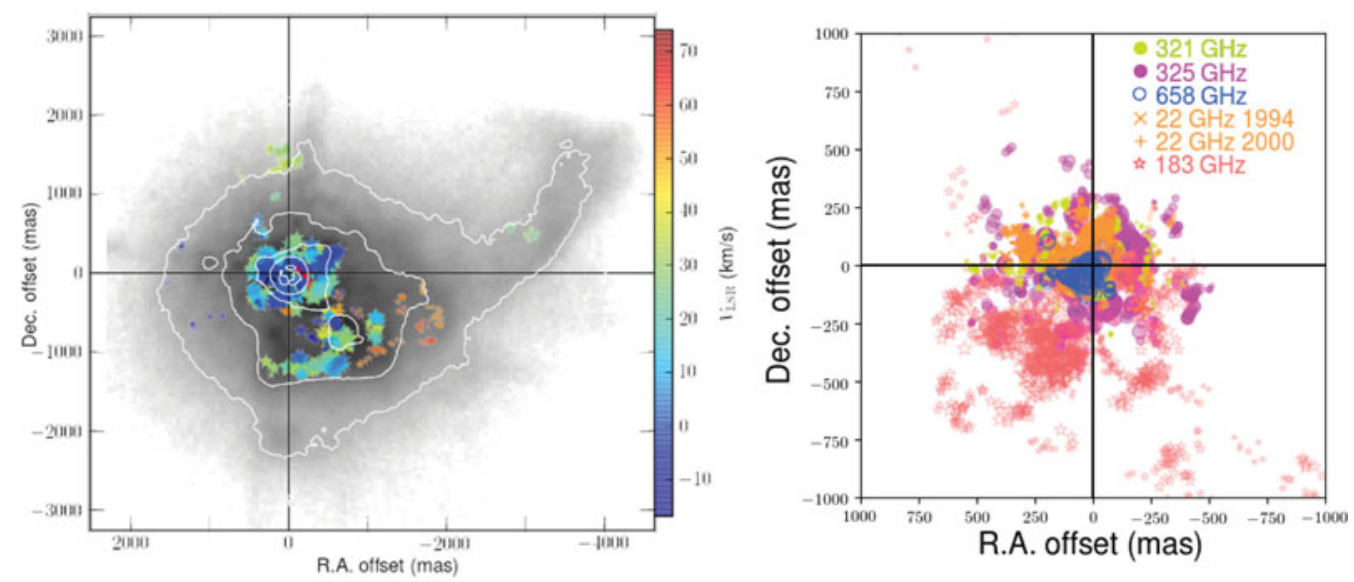

Figure 2. (left) $183 \mathrm{GHz}$ maser components, shaded by velocity, overlaid on an HST scattered light image. (right) Masers within $1^{\prime \prime}$ of the star at $(0,0)$; colour and shape represents species.
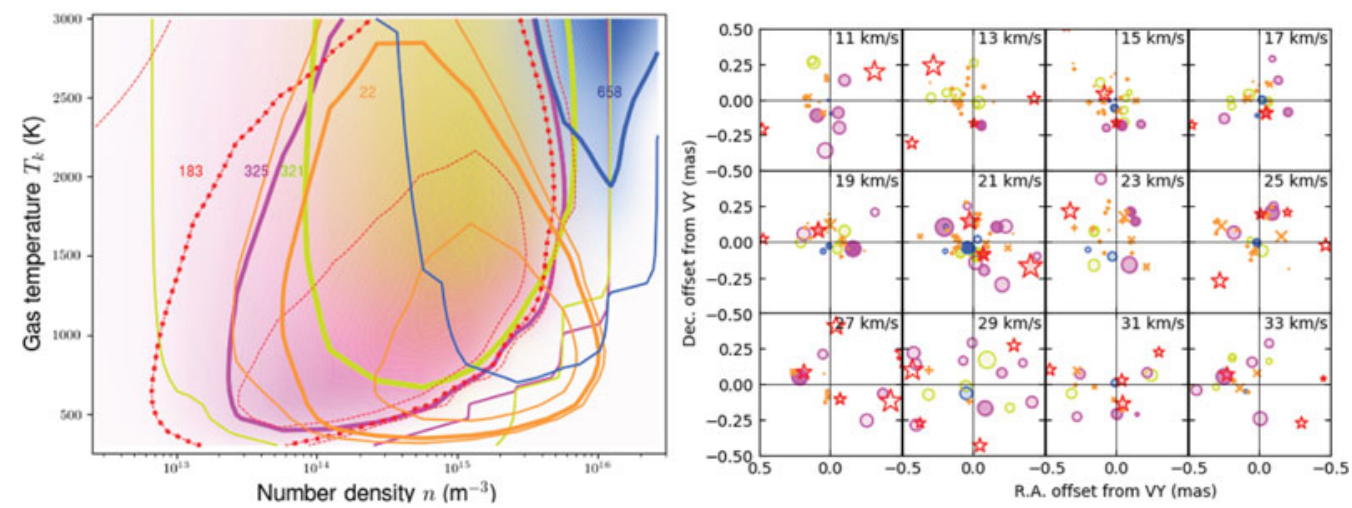

Figure 3. (left) Maser optical depth $\tau$ for 5 lines. Heavy contours show $50 \%$ maximum inversion. The lowest contour represents the observational sensitivity limit. $\tau$ is also shown by a transparent shade for 321, 325 and $658 \mathrm{GHz}$ masers. (right) (sub-)mm maser clump positions within 0." 5 of the star, colour and shape represents species, symbol size proportional to clump size.

On large scales, the $183-\mathrm{GHz}$ masers trace the distribution of small, cool dust grains seen in scattered light with the HST (Fig. 2), consistent with the prediction that their excitation extends to cool, low-density conditions. Maser distributions within $1^{\prime \prime}$ of the star are broadly consistent with expectations, in that the $658-\mathrm{GHz}$ emission is found closest to the star, with $321-, 22-$ and $325-\mathrm{GHz}$ at increasing radii (Fig. 2 (right)). The biggest surprise is that the $658-\mathrm{GHz}$ emission extends $>200$ mas from the star, well outside the $\sim 70$ mas radius of most dust formation. This could be due to shock heating as the wind collides with dust clump C, 328 mas SE of the star (O'Gorman et al. 2015).

All the masers lie in broad, overlapping, clumpy shells and we compared the $\sim 10$ au-scale distribution with model predictions, looking at $2 \mathrm{~km} \mathrm{~s}^{-1}$ velocity bins. We only considered emission within $V_{\star} \pm 12 \mathrm{~km} \mathrm{~s}^{-1}$ due to the asphericity of the VY CMa CSE making it difficult to convert $V_{\mathrm{LSR}}$ to position along the line of sight, and thus to determine whether apparently cospatial or adjacent emission at a particular velocity was actually at the same depth. We did not use the $22 \mathrm{GHz}$ data as the observations closest in time were taken under poor conditions. We tailored the Gray et al. (2016) models to VY CMa by taking the average angular size of the clumps for each line as the propagation 
depth. Fig 3 (left) shows where different masers are expected to co-propagate or occur alone. Fig 3 (right) shows the observed distribution of 183, 321, 325 and $658 \mathrm{GHz}$ maser clumps in the inner arcsec. The symbol size represents clump size. We matched pairs of clumps of different lines if they overlapped in velocity by half the maximum $V_{\text {LSR }}$ span of a clump (i.e. $50 \%$ overlap) and by half the sum of their angular sizes (i.e. just touching, to allow for aligment uncertainties of order $R_{\star}$ ).

Given the large areas of overlap in Fig. 3, we were surprised to find only 13 groups of 2 or more masers lines appearing to co-propagate (out of $\sim 70-170$ features per line). The required ranges of $n$ and $T_{\mathrm{k}}$ were determined from Fig. 3 for the combinations detected. Matsuura et al. (2014) and Decin et al. (2006) derived 1D models for VY CMa. Our results show that the wind acceleration is more gradual than predicted and reaches a higher terminal velocity than their models' gas velocity, possibly due to dust evolution or better momentum coupling in dense clumps. We obtained values of $n>50 \times$ higher close to the star, consistent with maser clumping, with $n$ for 183 - and $325-\mathrm{GHz}$ masers at closer to the average at $\geqslant 400$ mas radius, possibly from inter-clump gas. The $T_{\mathrm{k}}$ profile roughly supported the Decin model including the effects of a variable mass loss rate.

\section{Future work}

We have demonstrated the potential of multi-line imaging of $\mathrm{H}_{2} \mathrm{O}$ masers to constrain physical conditions on ten-au scales, covering the dust formation and wind acceleration regions of an RSG CSE. The model output will be refined using conditions closer to those in VY CMa. A constant dust temperature of $50 \mathrm{~K}$ was used here; a higher value closer to the star would be more realistic. We will also investigate the effect of using $f_{\mathrm{nH} 2 \mathrm{O}}$ of $2 \times 10^{-4}$ (Matsuura et al. 2014 ) or $1.1 \times 10^{-3}$ (Decin et al. 2006). However, two major problems are the uncertainty in estimating the depth of individual maser clumps and their location along the line of sight. At present, we assume that all clumps are spherical so the depth is the same as the angular size in order to estimate the relative maser $\tau$ from the flux density and propagation length, but some of the brightest clouds have small angular sizes, leading to unrealistically high $\tau$, probably because they have a much greater depth than assumed. The aspect ratio of clouds is constrained by the relationship between beaming angle $\Omega$ and flux density (Section 1), distinguishing between (on average) spherical clouds and shocked slabs. This requires observations using $\sim 8-16 \mathrm{~km}$ ALMA baselines. Accurate $3 \mathrm{D}$ modelling of the CSE as a whole requires a less aspherical target such as VX Sgr.

\section{References}

Bowers, P. F., Claussen, M. J., \& Johnston, K. J. 1993, AJ, 105, 284

Choi, Y. K., Hirota, T., Honma, M., et al. 2008, PASJ, 60, 1007

Cooke B. \& Elitzur M. 1985, ApJ, 295, 175

Decin, L. et al. 2006 A $E A$ 456, 549

Elitzur, M., Hollenbach, D. J., \& McKee, C. F. 1992, ApJ, 394, 221

Gray, M. D. et al. 2016, MNRAS, 456, 374

Humphreys, R. M., Helton, L. A., \& Jones, T. J. 2007, AJ, 133, 2716

Matsuura et al. 2014, MNRAS 437, 532

Marti-Vidal, I., Vlemmings, W. H.T., Carozzi, T. et al. 2016, https://bulk.cv.nrao.edu/almadata/sciver/VYCMaBand5/VYCMa_Band5_PolCalibrationInformation.pdf O'Gorman, E. et al. 2015, A\& A, 573, L10

Richards, A. M. S., Yates, J. A., \& Cohen, R. J. 1998, MNRAS 299, 319

Richards, A. M. S., Elitzur, M., \& Yates, J. A. 2011, A\& $A, 525,56$

Richards, A. M. S. et al. 2012, A $₫ A$ 546, 16

Richards, A. M. S. et al. 2014, A\&A, 572, L9

Wittkowski, M., Hauschildt, P. H., Arroyo-Torres, B., \& Marcaide, J. M. 2012, A\& $A$, 540, L12 